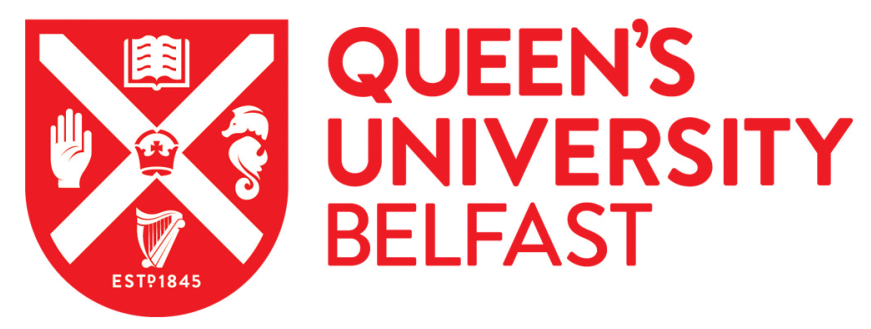

\title{
Superluminous supernova progenitors have a half-solar metallicity threshold
}

Chen, T. W., Smartt, S. J., Yates, R. M., Nicholl, M., Krühler, T., Schady, P., Dennefeld, M., \& Inserra, C. (2017). Superluminous supernova progenitors have a half-solar metallicity threshold. Monthly Notices of the Royal Astronomical Society, 470(3), 3566-3573. https://doi.org/10.1093/mnras/stx1428

Published in:

Monthly Notices of the Royal Astronomical Society

Document Version:

Publisher's PDF, also known as Version of record

Queen's University Belfast - Research Portal:

Link to publication record in Queen's University Belfast Research Portal

\section{Publisher rights}

Copyright 2017 The Authors

Published by Oxford University Press on behalf of the Royal Astronomical Society. This work is made available online in accordance with the publisher's policies. Please refer to any applicable terms of use of the publisher.

\section{General rights}

Copyright for the publications made accessible via the Queen's University Belfast Research Portal is retained by the author(s) and / or other copyright owners and it is a condition of accessing these publications that users recognise and abide by the legal requirements associated with these rights.

Take down policy

The Research Portal is Queen's institutional repository that provides access to Queen's research output. Every effort has been made to ensure that content in the Research Portal does not infringe any person's rights, or applicable UK laws. If you discover content in the Research Portal that you believe breaches copyright or violates any law, please contact openaccess@qub.ac.uk. 


\title{
Superluminous supernova progenitors have a half-solar metallicity threshold
}

\author{
Ting-Wan Chen, ${ }^{1,2 \star}$ Stephen J. Smartt, ${ }^{2}$ Rob M. Yates, ${ }^{1}$ Matt Nicholl, ${ }^{3}$ \\ Thomas Krühler, ${ }^{1}$ Patricia Schady, ${ }^{1}$ Michel Dennefeld ${ }^{4}$ and Cosimo Inserra ${ }^{2}$ \\ ${ }^{1}$ Max-Planck-Institut für Extraterrestrische Physik, Giessenbachstraße 1, D-85748 Garching, Germany \\ ${ }^{2}$ Astrophysics Research Centre, School of Maths and Physics, Queen's University Belfast, Belfast BT7 1NN, UK \\ ${ }^{3}$ Harvard-Smithsonian Center for Astrophysics, 60 Garden Street, Cambridge, MA 02138, USA \\ ${ }^{4}$ Institut d'Astrophysique de Paris, CNRS, and Universite Pierre et Marie Curie, 98 bis Boulevard Arago, F-75014 Paris, France
}

Accepted 2017 June 7. Received 2017 June 7; in original form 2016 May 18

\begin{abstract}
Host galaxy properties provide strong constraints on the stellar progenitors of superluminous supernovae. By comparing a sample of 19 low-redshift $(z<0.3)$ superluminous supernova hosts to galaxy populations in the local Universe, we show that sub-solar metallicities seem to be a requirement. All superluminous supernovae in hosts with high measured gas-phase metallicities are found to explode at large galactocentric radii, indicating that the metallicity at the explosion site is likely lower than the integrated host value. We found that superluminous supernova hosts do not always have star formation rates higher than typical star-forming galaxies of the same mass. However, we confirm that high absolute specific star formation rates are a feature of superluminous supernova host galaxies, but interpret this as simply a consequence of the anticorrelation between gas-phase metallicity and specific star formation rate and the requirement of on-going star formation to produce young, massive stars greater than $\sim 10-20 \mathrm{M}_{\odot}$. Based on our sample, we propose an upper limit of $\sim 0.5 \mathrm{Z}_{\odot}$ for forming superluminous supernova progenitors (assuming an N2 metallicity diagnostic and a solar oxygen abundance of 8.69). Finally, we show that if magnetar powering is the source of the extreme luminosity, then the required initial spins appear to be correlated with metallicity of the host galaxy. This correlation needs further work, but if it applies, it is a powerful link between the supernova parameters and nature of the progenitor population.
\end{abstract}

Key words: stars: magnetars - supernovae: general - supernovae: individual (SN 2011ke, SN 2012il, SN 2015bn, Gaia16apd) - galaxies: abundances - galaxies: dwarf.

\section{INTRODUCTION}

The host galaxies of superluminous supernovae (SLSNe) Type I are generally faint dwarf galaxies (Neill et al. 2011) that tend to have low gas-phase metallicities (Stoll et al. 2011; Chen et al. 2013; Lunnan et al. 2013), and high specific star formation rates (sSFR $\equiv$ $\mathrm{SFR} / M_{*}$ ) (Leloudas et al. 2015). There is currently a debate in the literature as to which of these two properties is the key requirement for SLSN-progenitor formation. Evidence for low-metallicity being the primary driver comes from Chen et al. (2013), who found the host galaxy of SN $2010 \mathrm{gx}$ to have a very low oxygen abundance of $0.06 \mathrm{Z}_{\odot}\left(12+\log (\mathrm{O} / \mathrm{H})=7.45 \pm 0.10\right.$ on the $T_{\mathrm{e}}$ scale $)$. The highquality host galaxy spectrum enabled the detection of the [O III] $4363 \AA$ line, providing a reliable 'direct' method estimate of the oxygen abundance for the first time for any such SLSN host. This

^E-mail: jchen@mpe.mpg.de is still the lowest metallicity for any supernova (or low-redshift gamma-ray burst, GRB) host galaxy measured to date. It is known from theory and observation that low-metallicity environments lead to more compact, faster rotating massive stars with weaker stellar winds (e.g. Yoon, Langer \& Norman 2006; Martayan et al. 2007; Mokiem et al. 2007; Hunter et al. 2008; Brott et al. 2011). This may favour magnetar formation, which is a viable proposed central engine model for SLSNe as proposed by Kasen \& Bildsten (2010) and Woosley (2010). The application of this model shows good quantitative agreement with observations of SLSNe (e.g. Inserra et al. 2013; Nicholl et al. 2013) and an SN associated with an ultra-long GRB (Greiner et al. 2015). In the first of the large sample papers, Lunnan et al. (2014) proposed that the hosts of Type I SLSNe share the same low-metallicity (sub-solar abundance) preference as long-duration GRB (LGRB) host galaxies. In fact, Type I SLSNe could require even lower metallicity environments than LGRBs (Chen et al. 2015). That the host galaxies of Type I SLSNe are consistently fainter than those of LGRBs is supported by the HST 
imaging (Angus et al. 2016). Vreeswijk et al. (2014) showed that interstellar medium (ISM) absorption features may also indicate different environments for SLSNe and GRBs.

Leloudas et al. (2015) proposed that high SSFR is the primary driver to produce SLSNe, and they found that half of their SLSN hosts are extreme-emission-line galaxies (i.e. galaxies exhibiting emission lines with EW >100 Aं; e.g. Calabrò et al. 2017). They suggested that the progenitors of SLSNe constitute the first generation of stars to explode in a starburst, therefore being even younger and more massive than the progenitors of LGRBs. In contrast, Lunnan et al. (2015) have instead suggested that SLSN Type I progenitors are older and less massive stars than those of LGRBs. This is based on the locations of Type I SLSNe within their hosts, which show less of a preference for the brightest regions of the host galaxy when compared to LGRBs. Another potential argument is that some SLSN host galaxies have been observed with possible high metallicities, which questions the proposal that low metallicity is a key requirement. For example, one of the most metal-rich host galaxies of an SLSN Type I is MLS121104 (Lunnan et al. 2014), with $12+\log (\mathrm{O} / \mathrm{H})=8.8\left(R_{23}\right.$ scale, Kobulnicky \& Kewley 2004; hereafter KK04). However, the SN location is clearly offset from the host centre, and further investigation is required to determine if this is indeed the host, or if the metallicity at the SN position is the same as that of the observed galaxy. Also, the metallicity estimate needs verification with the detection of the [O III] $\lambda 4363 \AA$ line. In this work, we found that the host galaxy of MLS121104 has $12+$ $\log (\mathrm{O} / \mathrm{H})=8.30 \pm 0.02$ using the N2 scale of Pettini \& Pagel (2004, hereafter PP04).

We note that SSFR itself cannot be a direct physical cause of SLSN progenitor formation, as it is not possible for a star-forming region to identify the total SFR or integrated star formation history (i.e. $M_{*}$ ) of the whole host galaxy. Therefore, for this interpretation to be valid, sSFR must instead be indicative of a more physical property. For example, a high sSFR could indicate a young stellar population, which could therefore contain massive stars capable of forming SLSNe (e.g. as proposed by Leloudas et al. 2015). Alternatively, a top-heavy stellar initial mass function (IMF) has been proposed for star-bursting regions in ultra-compact dwarf galaxies (Dabringhausen et al. 2012; Marks et al. 2012). This would allow an increased number of massive stars to form in these environments, hence causing the preference for SLSN to occur in high-sSFR galaxies. We would also note that adjusting the IMF has no observational support from resolved studies of massive stars in Local Group or local Universe galaxies, across factors of 5-10 in metallicity (see the reviews and references therein of Elmegreen 2006; Bastian, Covey \& Meyer 2010; Fumagalli, da Silva \& Krumholz 2011; Massey 2011).

In this work, we systematically compare the metallicity and star formation properties of a sample of 19 SLSN Type I host galaxies against star-forming galaxies in the local Universe, in order to better determine which is the most important parameter for producing SLSN progenitors.

\section{OBSERVATIONAL DATA}

\subsection{SLSN host sample}

We have compiled a sample of Type I SLSNe host data either in our possession (Chen et al. 2013, 2015; Chen 2015; Chen et al. 2017) or published in the literature (Lunnan et al. 2014; Leloudas et al. 2015) for all objects below $z<0.3$. In this work, we have supplemented this sample with two additional host galaxy metallicity measurements taken from available late-time SN spectra (SN 2015bn, Gaia16apd), and upper limits on the host galaxy SFR for an additional two hosts (LSQ12dlf, SN 2013dg). This makes up a sample of 19 low- $z$ SLSN Type I host galaxies. The SFR (from the $\mathrm{H} \alpha$ luminosity) and stellar mass have been corrected to the same IMF from Chabrier (2003). All stellar masses are taken from Schulze et al. (2016), and they are thus all derived in a consistent way (except for Gaia16apd, which we measured ourselves but using the same galaxy fitting templates as in Schulze et al. 2016). We have propagated all errors to obtain an overall uncertainty for each property. We also measure a detection limit for the $\mathrm{H} \alpha$ line where there is no reported detection to estimate an upper limit for the SFR in these cases. Those host properties are summarized in Table 1 . We only consider SLSN Type I hosts below $z=0.3$, with the argument that there should be only minor stellar-mass and metallicity evolution between $z=0.1$ (the minimum redshift in our sample) and $z=0.3$. The difference in the cosmic star formation rate density between $z \simeq 0.1$ and $\simeq 0.3$ using equation 15 in Madau \& Dickinson (2014) is only $0.011 \mathrm{M}_{\odot} \mathrm{yr}^{-1} \mathrm{Mpc}^{-3}$. The evolution in the gas-phase metallicity of star-forming galaxies between $z \simeq 0.08$ and 0.29 is less than 0.1 dex at $\log \left(M_{*} / \mathrm{M}_{\odot}\right) \sim 9.0$ (Zahid et al. 2014).

\subsection{Comparison galaxy sample}

Unlike previous studies, which primarily focused on comparisons between SLSN hosts and GRB hosts, in this work, we compare our SLSN sample with a Spitzer Local Volume Legacy (LVL) survey from Cook et al. (2014), which consists of nearby galaxies within $11 \mathrm{Mpc}$ (see Lee et al. 2009), as well as the Sloan Digital Sky Survey (SDSS) star-forming sample of Yates et al. (2012), which includes $\sim 110000$ galaxies with redshifts in the range $0.005<z<0.25$. Unlike the LVL sample, the SDSS sample is not complete, but its advantage is that it is at the same redshift regime as our SLSN host galaxy sample, and thus cosmic redshift evolution effects should not be a problem. This allows us to better identify the key property required to produce Type I SLSNe. ${ }^{1}$ We cross match the LVL sample with an $\mathrm{H} \alpha$ imaging survey (i.e. narrow-band photometry around the $\mathrm{H} \alpha$ line) of galaxies within $11 \mathrm{Mpc}$ sample taken from Kennicutt et al. (2008). This leaves us with a final sample of 204 galaxies that includes both dwarf and giant irregular/spiral star-forming galaxies, spanning a wide luminosity range of $-9.6<\mathrm{M}_{B}<-20.7$.

Kennicutt et al. (2008) give the $\mathrm{H} \alpha$ luminosities for those galaxies, which we use to estimate the SFR. These luminosities are corrected for Milky Way reddening but not for internal extinction. Therefore, we have made our own internal dust extinction corrections using the Balmer decrement $(\mathrm{H} \alpha / \mathrm{H} \beta)$ for 13 galaxies (Moustakas et al. 2010) within the $11 \mathrm{Mpc}$ catalogue finding a correlation between their average $A_{V}$ and their observed galaxy $\mathrm{H} \alpha$ luminosities of $A_{V}=0.9445 \times \log \mathrm{L}_{H \alpha}-36.536$ (with a correlation of 0.70 and a $1 \sigma$ scatter in $A_{V}$ of $0.6 \mathrm{mag}$ ). We use this relation to obtain extinctions for the remainder of the Kennicutt sample. Negative values of $A_{V}$ are set to zero. We then employ the calibration of Kennicutt (1998) (which assumes an IMF of Salpeter 1955) to estimate galaxy SFR from the extinction-corrected $\mathrm{L}_{H \alpha}$, and apply a further correction to convert to a Chabrier IMF by multiplying by a factor of 0.63 .

\footnotetext{
${ }^{1}$ While this paper was in preparation, a paper using similar methods, but different sample was released as a preprint (Perley et al. 2016). However, we note that our work was carried out independently.
} 
Table 1. The low-redshift $(z<0.3)$ SLSN Type I host galaxy properties. The SFR (from dust-corrected $\mathrm{H} \alpha$ luminosity) and stellar mass are calculated assuming a Chabrier IMF.

\begin{tabular}{|c|c|c|c|c|c|}
\hline Name & Redshift & $\begin{array}{c}\text { N2 (PP04) } \\
(12+\log (\mathrm{O} / \mathrm{H}))\end{array}$ & $\begin{array}{c}\log \text { stellar mass } \\
\left(\mathrm{M}_{\odot}\right)\end{array}$ & $\begin{array}{c}\mathrm{SFR} \\
\left(\mathrm{M}_{\odot} \mathrm{yr}^{-1}\right)\end{array}$ & $\begin{array}{c}\text { sSFR } \\
\left(\mathrm{Gyr}^{-1}\right)\end{array}$ \\
\hline PTF10hgi & 0.098 & $8.38(0.05)^{a}$ & $7.58_{-0.31}^{+0.29}$ & $0.04(0.03)^{a}$ & $1.05(0.79)$ \\
\hline SN 2010kd & 0.101 & $8.07(0.05)^{a}$ & $7.30_{-0.29}^{+0.25}$ & $0.07(0.01)^{a}$ & $3.51(0.52)$ \\
\hline Gaia16apd & 0.101 & $8.05(0.04)^{b}$ & $7.40_{-0.80}^{+0.90 *}$ & $0.35(0.01)^{b}$ & $13.93(1.74)$ \\
\hline PTF12dam & 0.107 & $8.10(0.02)^{c}$ & $8.89_{-0.30}^{+0.15}$ & $4.83(0.09)^{a}$ & $6.22(0.16)$ \\
\hline SN 2015bn & 0.114 & $8.18(0.02)^{d}$ & $7.50_{-0.35}^{+0.38}$ & $0.03(0.00)^{d}$ & $0.95(0.05)$ \\
\hline SN 1999as (SN location) & 0.127 & $<8.29^{a}$ & - & $0.04(0.02)^{a}$ & - \\
\hline SN 2007bi & 0.128 & $8.20(0.06)^{a}$ & $7.92_{-0.21}^{+0.20}$ & $0.01(0.00)^{e}$ & $0.12(0.01)$ \\
\hline SN 2011ke & 0.143 & $7.82(0.11)^{f}$ & $7.50_{-0.18}^{+0.20}$ & $0.39(0.01)^{f}$ & $12.25(0.45)$ \\
\hline SSS120810 & 0.156 & $<8.23^{a}$ & $7.42_{-0.17}^{+0.21}$ & $0.06(0.04)^{a}$ & $2.28(1.52)$ \\
\hline LSQ14an & 0.163 & $7.98(0.04)^{f}$ & $8.54_{-0.17}^{+0.13}$ & $1.01(0.02)^{f}$ & $2.92(0.07)$ \\
\hline SN 2012il & 0.175 & $8.09(0.02)^{f}$ & $8.20_{-0.17}^{+0.18}$ & $0.32(0.01)^{f}$ & $2.01(0.08)$ \\
\hline PTF11rks & 0.192 & $8.42(0.15)^{g}$ & $8.96_{-0.14}^{+0.12}$ & $0.31(0.03)^{h}$ & $0.34(0.03)$ \\
\hline SN 2010gx & 0.23 & $7.97(0.06)^{i}$ & $7.97_{-0.13}^{+0.14}$ & $0.41(0.01)^{i}$ & $4.42(0.13)$ \\
\hline SN 2011kf & 0.245 & $<8.29^{a}$ & $7.58_{-0.22}^{+0.19}$ & $0.15(0.05)^{a}$ & 3.95 (1.32) \\
\hline LSQ12dlf & 0.250 & - & $7.56_{-0.34}^{+0.33}$ & $<0.004^{j}$ & $<0.11$ \\
\hline LSQ14mo & 0.256 & $8.18(0.05)^{k}$ & $7.89_{-0.19}^{+0.15}$ & $0.06(0.01)^{k}$ & $0.81(0.13)$ \\
\hline PTF09cnd & 0.258 & $8.24(0.06)^{a}$ & $7.87_{-0.21}^{+0.20}$ & $0.21(0.05)^{a}$ & $2.83(0.68)$ \\
\hline SN 2013dg & 0.265 & - & $7.09_{-0.70}^{+0.82}$ & $<0.003^{j}$ & $<0.24$ \\
\hline MLS121104 & 0.303 & $8.30(0.02)^{g}$ & $9.27_{-0.24}^{+0.25}$ & $0.79(0.02)^{h}$ & $0.43(0.02)$ \\
\hline
\end{tabular}

Note: All stellar mass are from Schulze et al. (2016), except Gaia16apd*, which we measured ourselves but using the same galaxy fitting templates as in Schulze et al. (2016).

${ }^{a}$ Leloudas et al. (2015);

${ }^{b}$ measured SN spectrum from Nicholl et al. (in preparation);

${ }^{c}$ Chen et al. (2015);

${ }^{d}$ measured SN spectrum from Jerkstrand et al. (2017);

${ }^{e}$ Young et al. (2010);

${ }^{f}$ Chen (2015);

${ }^{g}$ adopted flux measurement from Lunnan et al. (2014);

${ }^{h}$ Lunnan et al. (2014);

${ }^{i}$ Chen et al. (2013);

${ }^{j}$ measured SN spectrum from Nicholl et al. (2014);

${ }^{k}$ Chen et al. (2017).

We take the stellar masses derived from Spitzer $3.6 \mu \mathrm{m}$ measurements from Cook et al. (2014), who assumed a mass-to-light ratio of 0.45 (McGaugh \& Schombert 2015). McGaugh \& Schombert (2015) found that their analysis is consistent with results based on galaxy SED fitting that assume a Kroupa or Chabrier IMF, and therefore a direct comparison of the Cook et al. (2014) stellar masses with our SLSN sample is justified.

Kennicutt et al. (2008) also provide the ratio $[\mathrm{N} \mathrm{II}] / \mathrm{H} \alpha$ from spectroscopic observations and from the correlation between $\left[\mathrm{N}\right.$ II] $/ \mathrm{H} \alpha$ and $M_{B}$, which we use to calculate metallicities via the $\mathrm{N} 2$ method of PP04. However, the ratio they provide is for [N II] $\lambda \lambda 6548,6583$, whereas the $\mathrm{N} 2$ scale uses only [N $\mathrm{NI}] \lambda 6583$. We estimated the ratio of $[\mathrm{N} \mathrm{II}] \lambda 6583$ and $\mathrm{H} \alpha$ by applying the theoretical ratio between the [N II] lines at $6548 \AA$ and $6583 \AA$ of $1: 3$, and then calculated their metallicities with the $\mathrm{N} 2$ scale.

\section{RESULTS}

\subsection{Metallicity versus SSFR}

Fig. 1 shows the relationship between SFR and metallicity (N2 method) for 19 low- $z$ SLSN Type I hosts from the literature (red

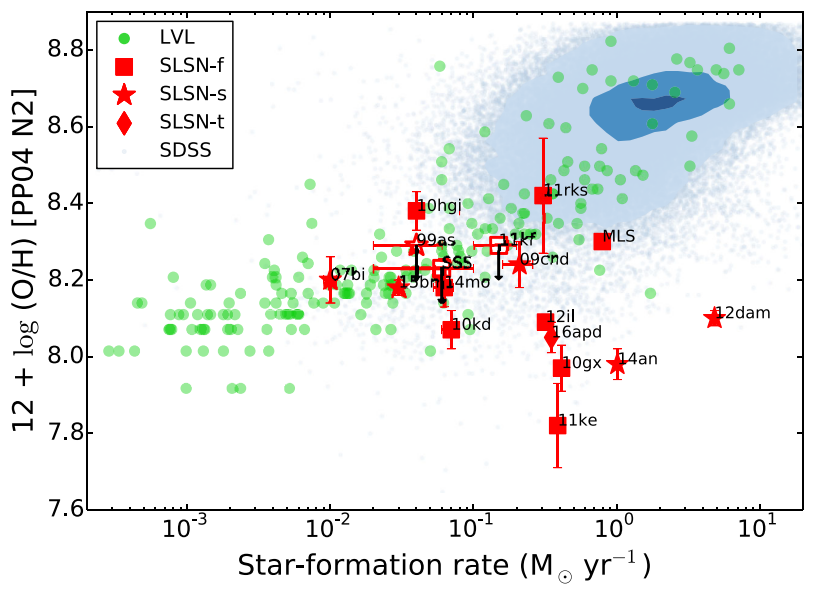

Figure 1. Star formation rate-metallicity relation for SLSN Type I host galaxies and the LVL and the SDSS galaxies.

points, see Section 2.1). The LVL (green) and SDSS (blue) samples of nearby galaxies are also shown for comparison. Type I SLSNe seem to be divided into two groups based on light-curve 


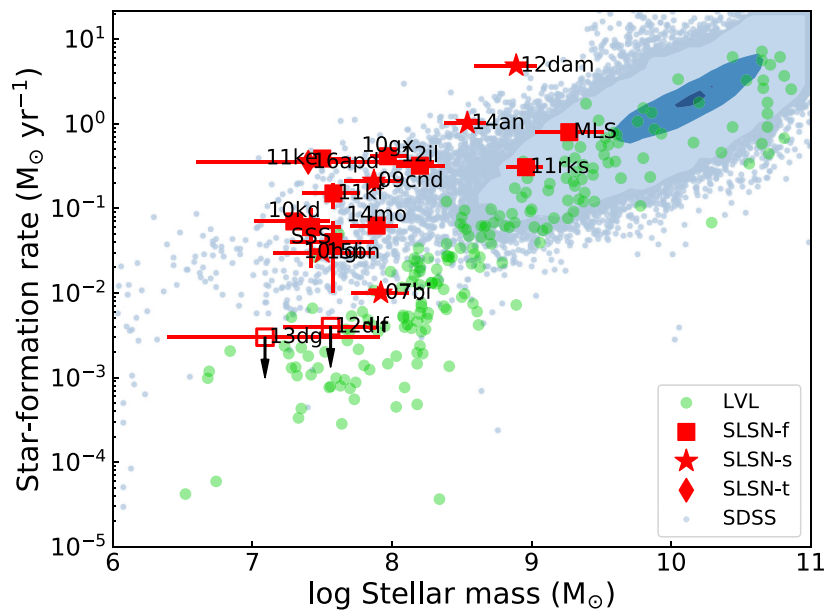

Figure 2. The stellar mass-SFR relation for SLSN Type I host galaxies and the LVL and the SDSS galaxies.

evolution. The majority have a fast rising and declining light curve (e.g. SN 2010gx), but a subset show a slow-evolving light curve (e.g. SNe 2007bi) (see Nicholl et al. 2015). Recently the light curve of Gaia16apd was found to be an intermediate case between the fast- and slow-declining SLSNe (Kangas et al. 2017), hence we refer it as a transition object. We use different markers (square for fast-decliners, star for slow-decliners and diamond for transition) to highlight these three sub-class of SLSNe, and we use the same markers throughout the paper. We use the PP04 N2 metallicity diagnostic for all SLSN hosts, and for the LVL and SDSS samples so that we can make a fair comparison of their relative metallicities. The N2 method is reliable in the low-metallicity regime where our SLSN hosts are located. We can see that the most star-forming SLSN hosts have significantly lower metallicities than local galaxies with similarly high SFRs. No SLSN host in our sample has a measured metallicity higher than $12+\log (\mathrm{O} / \mathrm{H}) \sim 8.4$, which is roughly half the solar oxygen abundance [assuming $12+\log (\mathrm{O} / \mathrm{H})_{\odot}=8.69$; Asplund et al. 2009. For comparison, Modjaz et al. (2011) measured oxygen abundances at the SN position of 12 normal Type Ic $\mathrm{SNe}$, and found that the metallicities of those hosts are all above 8.5 dex (PP04 O3N2 scale, which is similar to N2 scale).

Fig. 2 shows the relation between $M_{*}$ and SFR for the same three samples. The LVL and SDSS samples diverge at the low stellar mass regime. SLSN hosts are more star-forming than LVL galaxies of the same stellar mass. However, they have typical (or slightly elevated) SFRs for their stellar mass compared to the SDSS sample, and lower SFRs than the bulk of the overall star-forming population. Correspondingly, Fig. 3 shows that most SLSN hosts have high sSFR in an absolute sense (i.e. compared to the majority of the whole star-forming population in the SDSS). However, there are three hosts (SN 2007bi, LSQ12dlf and SN 2013dg) that exhibit lower specific SFRs than the rest of our SLSN-host sample. We note that the derived stellar mass values vary depending on the stellar population synthesis models assumed, even when the same IMF is used. For example, the stellar mass of SLSN hosts given in Schulze et al. (2016) is on average 0.2 dex lower than that in other literature (Perley et al. 2016) while comparing the same host galaxies, which introduces 2.5 times higher sSFR.

Similarly, Fig. 4 shows that most SLSN hosts have comparable or lower metallicities than local galaxies of the same stellar mass. These systems are qualitatively consistent with the low-redshift fundamental metallicity relation (FMR; Mannucci et al. 2010), which

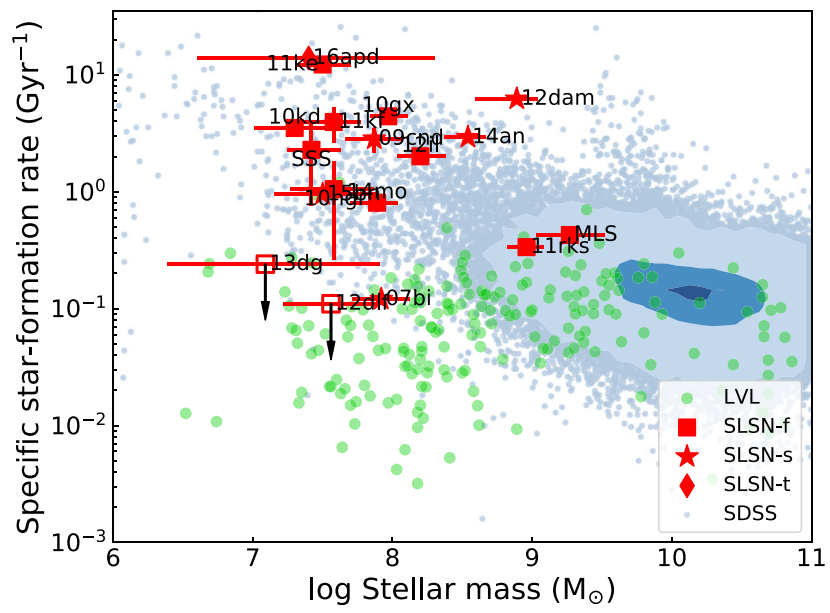

Figure 3. The stellar mass-SSFR relation for SLSN Type I host galaxies and the LVL and the SDSS galaxies. SLSN hosts typically have high sSFRs compared to the overall local star-forming population.

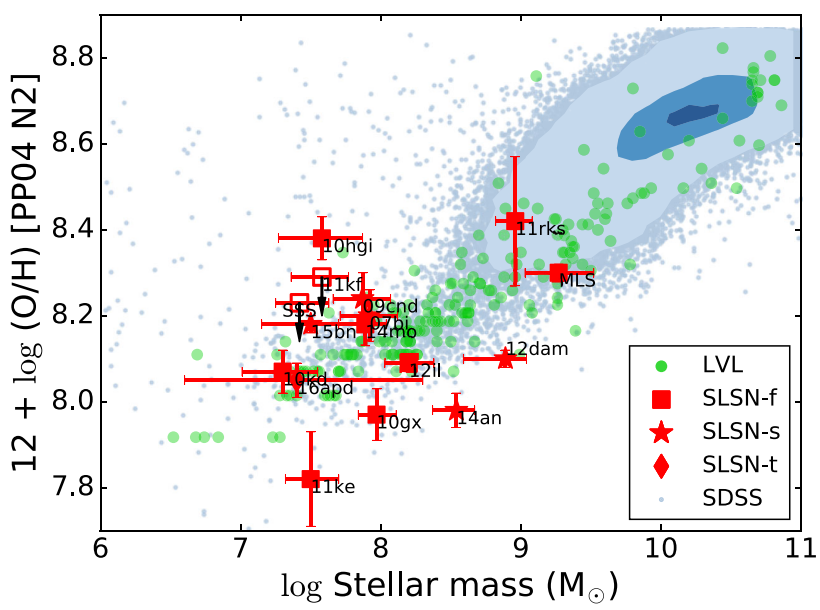

Figure 4. The stellar mass-metallicity relation for SLSN Type I host galaxies and the LVL and the SDSS galaxies. No SLSN hosts above $12+$ $\log (\mathrm{O} / \mathrm{H})>8.4$.

suggests an anticorrelation between SFR and metallicity at low $M_{*}$. However, two of our SLSN hosts, PTF10hgi and SN 2015bn (and possibly also PTF09cnd), have metallicities higher than typical galaxies of the same mass (though still below 8.4, using the N2 method). This demonstrates that SLSN only requires progenitors with a metallicity below some absolute value, regardless of the typical metallicities found in galaxies of the same stellar mass.

Turning our attention to the relative significance of low metallicity and high specific star formation rate, we present the metallicitysSFR relation in Fig. 5. This can be compared to the same relation presented by Perley et al. (2016) in their fig. 11. We can see that our SLSN hosts lie in the lower metallicity regime, and tend to have elevated sSFR compared to local star-forming galaxies. We note that the choice of metallicity diagnostic does not affect this conclusion. The parameter space in Fig. 5 has also been divided up into four quadrants, such that our SLSN sample lies exclusively in the high-sSFR, low-metallicity quadrant. Only 2.41 per cent of the total star formation occurs in the quadrant associated with SLSN hosts.

To investigate this further, in Fig. 6 we plot the cumulative distribution of SFR for SLSN hosts (red line), and the LVL sample (green 


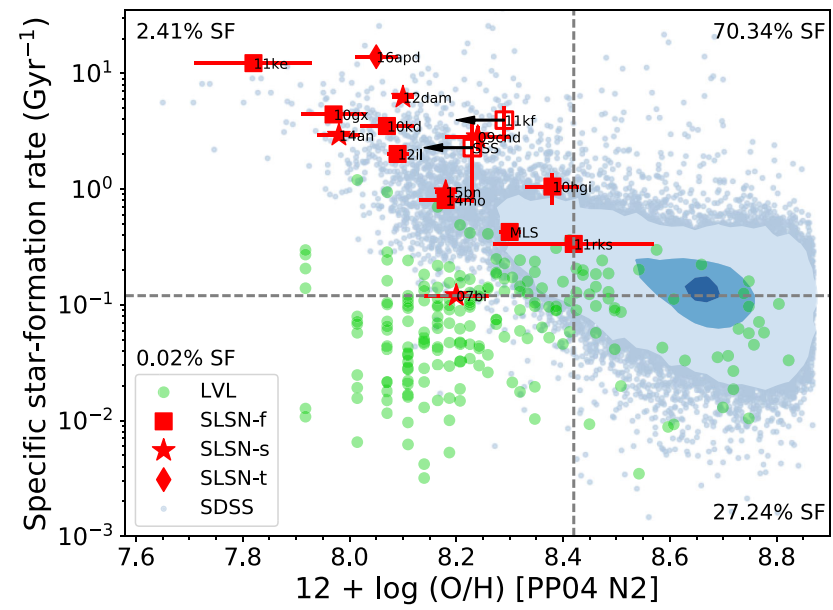

Figure 5. The metallicity-sSFR relation for SLSN Type I host galaxies, compared to that of local irregular/spiral galaxies from the LVL and the SDSS samples. SLSN Type I hosts are clearly seen to reside in galaxies with relatively high sSFR and metallicities below $12+\log (\mathrm{O} / \mathrm{H}) \sim 8.4$. The percentage of the total star formation rate in the local galaxy sample that occurs in each of the quadrants marked by the dashed lines is provided.
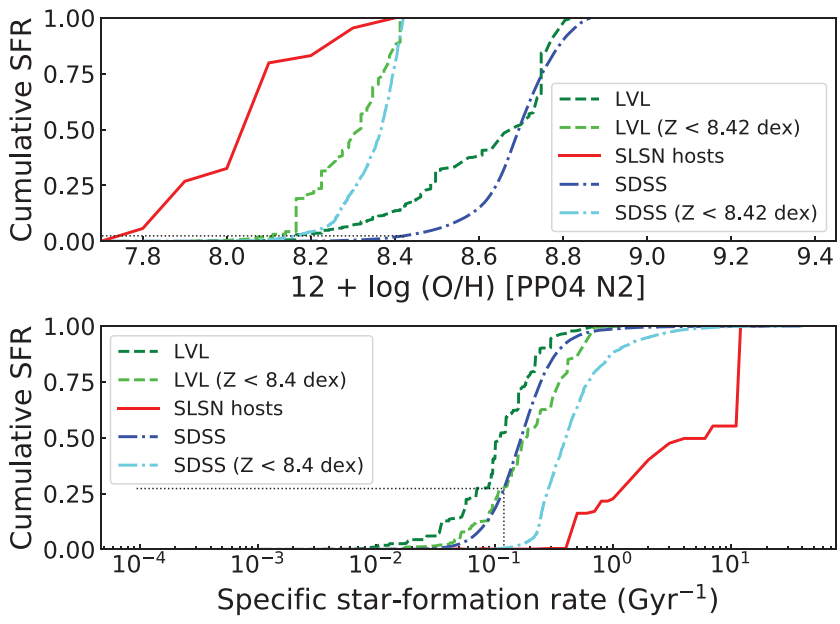

Figure 6. Cumulative SFR distribution of the LVL and the SDSS galaxies as a function of $\mathrm{N} 2$ ratio (i.e. [ $\mathrm{N} \mathrm{II}] / \mathrm{H} \alpha$ ). About 2.41 per cent of star formation occurs in the metallicity range where we observe SLSNe, suggesting that SLSN progenitors do not simply trace cosmic star formation.

colour lines) and the SDSS sample (blue colour lines) as a function of metallicity and sSFR. Only 7.85 per cent of the star formation in the local Universe occurs in galaxies with metallicities below 8.42 (i.e. with metallicities similar to those of SLSN hosts). Even when only considering these low-metallicity systems, SLSNe still favour lower metallicity star-forming regions than typically found in local galaxies. Similarly, SLSNe are preferentially found in the highest sSFR galaxies, even when compared only with the low-metallicity $(<8.42$ dex $)$ local population.

These two results suggest that SLSNe do not simply trace star formation in the local Universe, but instead prefer both low-metallicity and high-sSFR environments. The probability (assuming a binomial distribution) that all of the 19 low- $z$ SLSN Type I hosts are in the low-metallicity bin simply by chance is only $(7113 / 295545)^{19}=$ $1.77 \times 10^{-31}$.

The metallicity-sSFR parameter space has also been studied for the sample of $\sim 110000$ emission-line galaxies from the SDSS-

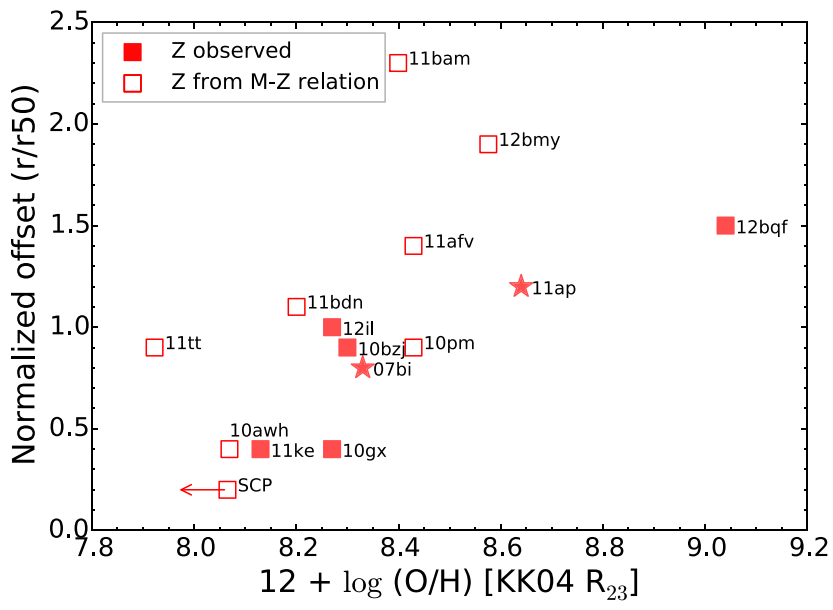

Figure 7. The metallicity-offset relation for SLSN Type I host galaxies from the Lunnan et al. $(2014,2015)$ sample. Hosts with known metallicities (filled red symbols) and with metallicities predicted from the massmetallicity relation (open red symbols) are shown. There is a correlation between the reported host metallicity (here measured with the KK04 $R_{23}$ method) and the galactocentric distance of the SLSN. Crucially, there are no systems in the bottom-right region of the plot, indicating that no SLSNe in our sample have exploded near the centres of high-metallicity galaxies.

DR7 by Yates \& Kauffmann (2014). Such samples can be used to make qualitative statements about the type of galaxies that seem to host SLSNe. Yates \& Kauffmann (2014) show that systems within the same range of sSFR and metallicity as our SLSN hosts have low stellar masses, high gas fractions and young ages, compared to typical star-forming galaxies in the local Universe (see their fig. 13). From analogy to the Munich semi-analytic model of galaxy evolution, L-GALAXIES, they also show that these systems' gas-phase metallicities should be lower than their stellar metallicities (see panel E of Fig. 3), indicating that recent accretion of low-metallicity gas is fuelling current star formation.

\subsection{Integrated metallicity versus SLSN offset}

Further evidence for the preference of SLSNe to occur in lowmetallicity environments is found in Fig. 7, where we plot metallicity versus galactocentric distance of the SLSN event for the sample of Lunnan et al. (2015), who measured the SN position normalized to the rest-frame UV half-light radius $\left(r_{50}\right)$ from $H S T$ images. This sample comprises seven host galaxies with metallicity measurements in the redshift range $0.12<z<0.65$, and eight host galaxies with only mass measurements in the redshift range $0.74<z<1.6$. To obtain metallicities for these higher redshift systems, we use a fit to the mass-metallicity relation for the lower redshift systems: $Z_{\mathrm{g}}(\mathrm{KK} 04)=0.37 \log \left(M_{*}\right)+5.32$ (with a correlation of 0.92 and a $1 \sigma$ scatter in $Z_{\mathrm{g}}$ of $\left.0.11 \mathrm{dex}\right)$. To account for the evolution in the mass-metallicity relation with cosmic time, we apply a shift in metallicity of -0.16 dex for host galaxies at redshifts around $z=$ 0.78 and -0.26 dex for those around $z=1.4$, following the evolution measured by Zahid et al. (2014) for galaxies of stellar mass $\sim 10^{9} \mathrm{M}_{\odot}$.

Fig. 7 shows a clear correlation between the host metallicity (in this case, measured with the KK04 $R_{23}$ method, since the $\mathrm{H} \alpha$ and [N II] lines are out of the observed wavelength range) and SLSN offset. More precisely, we can say that there are no SLSNe found near the centres of high-metallicity galaxies. Systems with disturbed morphologies may contain low-metallicity pockets of gas 
where SLSNe could form or the true host galaxy may be kinematically distinct. For example, in the case of LSQ14mo, Chen et al. (2017) found evidence for a possibly interacting system, with the SLSN lying in a smaller satellite galaxy that had a 0.4 dex lower metallicity than that of the main galaxy system. It is possible that an interacting galaxy system could induce gas flows triggering star formation in low-metallicity regions. Sánchez et al. (2014) showed that disc abundance gradients in spiral galaxies are common and universal when expressed in terms of effective radius, even down to absolute magnitudes of $M_{g} \simeq-18$. This may also be an indicator that abundance variations are in play in lower luminosity galaxies, and indicates that the metallicity at an SLSN site could be lower than the integrated measured value. Therefore, even in galaxies with high integrated metallicities (such as the host of PS1-12bqf at $z=0.52$ ), a sub-solar metallicity could still be present at the offset SLSN site. One observed example is SN 1999as, which has a large offset from its host centre (10.7 kpc; Leloudas et al. 2015). Although a high host metallicity of $12+\log (\mathrm{O} / \mathrm{H})=8.56(\mathrm{PP} 04 \mathrm{~N} 2)$ is measured, the metallicity measured at the SN location is more than $0.3 \mathrm{dex}$ lower $(12+\log (\mathrm{O} / \mathrm{H})<8.29)($ Lunnan et al. 2015).

\section{DISCUSSION}

As mentioned in Section 1, there are two dominant interpretations of current SLSN-host data. The first is that low metallicity is the main requirement for SLSN formation. The second is that high sSFR is instead the main requirement. From the data presented in Section 3, we cannot unambiguously distinguish between these two interpretations. Indeed, Fig. 5 demonstrates that both low metallicity and high sSFR are present in all of the SLSN host galaxies sampled here. However, in this work, we suggest that low metallicity is a fundamental cause, whereas high sSFR is only a consequence of the low metallicity.

Observations show a clear preference for SLSNe to occur in lowmetallicity environments, which implies low stellar mass host galaxies through the mass metallicity relation. We expect that SLSNe also require massive star progenitors, and thus they are more likely to arise in galaxies with elevated SFRs. Combining these two factors gives rise to the observed high sSFR. This interpretation is supported by the fact that there are many hosts that have weak (e.g. the host of SN 2007bi) or undetected $\mathrm{H} \alpha$ lines. For example, we estimate the detection limit of $\mathrm{H} \alpha$ fluxes from the SLSN spectra, and find the host of LSQ12dlf to have a detection limit of SFR < $0.004 \mathrm{M}_{\odot} \mathrm{yr}^{-1}$, the host of SN 2013dg to have a detection limit of SFR $<0.003 \mathrm{M}_{\odot} \mathrm{yr}^{-1}$ and the host of LSQ14bdq to have a detection limit of SFR $<0.05 \mathrm{M}_{\odot} \mathrm{yr}^{-1}$. These low SFR objects are not shown in figures due to the lack of information of other host properties (e.g. unknown host metallicities and stellar masses).

Leloudas et al. (2015) argued that mass is the key driver and that Type I SLSNe are the very first stars to explode in a starburst, and thus the progenitors are very massive stars (for specific object, see Thöne et al. 2015). Our data are not inconsistent with this interpretation, although we would point out that very high masses are not quantitatively constrained in either study. Along with the very young stars that provide the extreme emission line ratios, virtually all hosts have stellar populations up to a few tens of Myr old detected, as traced by the UV and optical continuum (Lunnan et al. 2014; Chen et al. 2015; Leloudas et al. 2015). The spatial resolutions that ground-based spectrographs probe at $z>0.1$ are typically more than $2 \mathrm{kpc}$, so a precise determination of the nature of progenitor stars is not yet possible. Distinguishing between progenitors of $\sim 10-20 \mathrm{M}_{\odot}$ and greater than $50 \mathrm{M}_{\odot}$ is not easily achieved

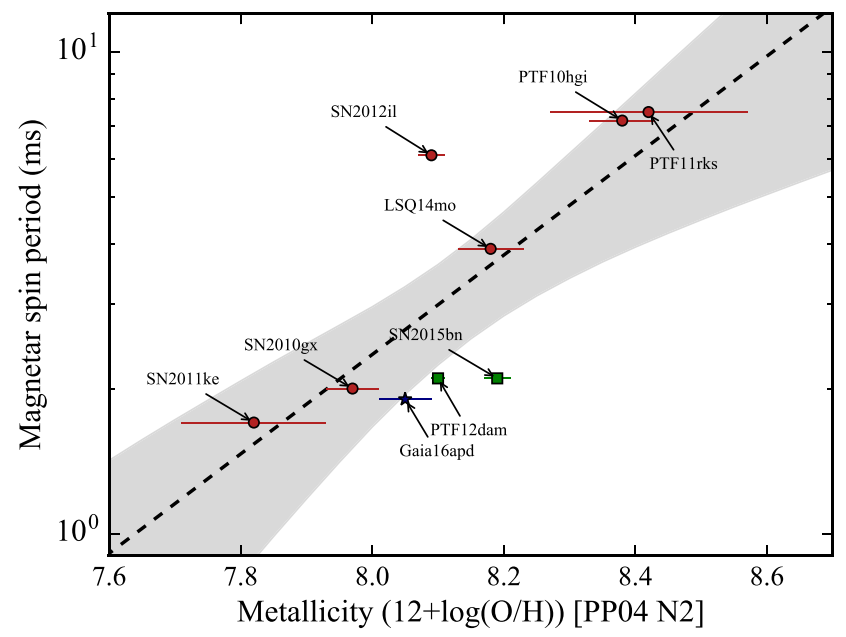

Figure 8. The host metallicity-magnetar spin-period relation. The red markers show the fast-decliners and the green markers show the slowly declining Type I SLSNe. The best fit shown by black dashed line and the grey area in which 68 per cent of all iteration are located.

with the data. SLSNe are rare, and occur only at a rate of about 1 in 10 000-20 000 of the core-collapse population (Quimby et al. 2011, 2013; McCrum et al. 2015). It may be that a combination of very high mass (as traced by high SSFR) and low metallicity is required. Or it could be that a high SSFR is just a consequence of star formation in low-metallicity environments.

Alternative scenarios that could produce low gas-phase metallicities in the SLSN region, without requiring low $M_{*}$, are (i) significant infall of pristine gas on to a previously higher metallicity host galaxy and (ii) variations in metallicity within a host itself (e.g. lowmetallicity pockets or global metallicity gradients). Crucially, both these scenarios are consistent with the presence of a low integrated sSFR. Therefore, with a larger sample of SLSN host galaxies with measured sSFRs, we could break the degeneracy discussed above and confirm our interpretation that low metallicity is the key driver.

\subsection{A possible link between progenitor metallicity and magnetar spin}

The identification of metallicity as the key parameter for forming Type I SLSNe has important implications for determining the power source underlying the explosion itself. As discussed in Section 1, the magnetar model is one of the preferred scenarios for producing SLSNe. Any link between galaxy environment and parameters of the powering mechanism would help elucidate not only progenitor star properties (e.g. mass or metallicity) but also the underlying mechanism producing SLSNe.

We show a possible correlation between host metallicity and magnetar spin-down period in Fig. 8 using the available data for eight SLSNe and their hosts. The magnetar spin-down period is derived from the SLSN bolometric light curve and semi-analytic models for magnetar energy deposition (Inserra et al. 2013; Nicholl et al. 2013, 2015, see Table 2). The two quantities are truly independent measurements. A simple Spearman rank correlation analysis returns a coefficient $\rho=0.85$. This corresponds to a null-hypothesis probability, that the observed correlation is the result of a statistical fluctuation, of $p=0.003$. This is an unexpectedly clear correlation between two quite independent observational properties.

However, measurement uncertainties play an important role and we hence test the significance of the observed correlation 
Table 2. Magnetar model parameters for nine Type I SLSNe. We select SLSNe that have a full light-curve coverage before and after the peak brightness and a good spectral coverage to apply for $K$-correction. We also choose the same magnetar code in order to have a consistent fitting result.

\begin{tabular}{lccccr}
\hline Name & Sub-type & $\begin{array}{c}\mathrm{P} \\
(\mathrm{ms})\end{array}$ & $\begin{array}{c}\mathrm{B} \\
(14 \mathrm{G})\end{array}$ & $\begin{array}{c}\mathrm{M}_{\mathrm{ej}} \\
\left(\mathrm{M}_{\odot}\right)\end{array}$ & Reference \\
\hline SN 2011ke & fast & 1.7 & 6.4 & 8.6 & Inserra et al. (2013) \\
SN 2010gx & fast & 2.0 & 7.4 & 7.1 & Inserra et al. (2013) \\
Gaia16apd & transition & 1.9 & 2 & 4.8 & Nicholl et al. (2017) \\
PTF12dam & slow & 2.1 & 1.5 & 16 & Chen et al. (2015) \\
SN 2015bn & slow & 2.1 & 0.9 & 8.4 & Nicholl et al. (2016) \\
LSQ14mo & fast & 3.9 & 5.1 & 3.9 & Chen et al. (2017) \\
SN 2012il & fast & 6.1 & 4.1 & 2.3 & Inserra et al. (2013) \\
PTF10hgi & fast & 7.2 & 3.6 & 3.9 & Inserra et al. (2013) \\
PTF11rks & fast & 7.5 & 6.8 & 2.8 & Inserra et al. (2013) \\
\hline
\end{tabular}

using a sample of $10^{5}$ bootstrapped distributions. Here, we vary the host metallicity according to the measurement errors and the spin-down period $\left(P_{\mathrm{ms}}\right)$ by an assumed systematic error of $0.2 \mathrm{dex}$. We fit a linear relation to each of the data sets. The median of the resulting posterior distribution is obtained at $\log \left(P_{\mathrm{ms}}\right)=$ $-7.89+1.03 \times(12+\log (\mathrm{O} / \mathrm{H}))$. This best fit, and the area in which 68 per cent of all iterations are located, is shown in Fig. 8. No dependence of spin-down period on host metallicity is observed in only $p=0.045$ of all cases. A positive correlation is also found when using the $T_{e}$ and KK04 $R_{23}$ metallicity diagnostics, with errors consistent with the N2-metallicity based data, although the relations obtained are somewhat steeper and have a slightly larger scatter.

The relation between the magnetar spin-down period and the host metallicity indicates that faster rotating magnetars reside in more metal-poor environments. Theory predicts that low-metallicity massive stars are more compact and have a reduced mass-loss, which results in faster rotation (Ekström et al. 2012; Szécsi et al. 2015) This may lead to chemically homogeneous evolution that allows massive stars to retain more angular moment and thus rotate faster, even during the Wolf-Rayet stage (Yoon et al. 2006; Brott et al. 2011). Observationally, massive stars with SMC metallicity $\left(\sim 0.2 \mathrm{Z}_{\odot}\right)$ appear to rotate more rapidly than those with solar metallicity (Martayan et al. 2007; Hunter et al. 2008; RamírezAgudelo et al. 2013) and the difference may also be visible at LMC metallicities. Further work needs to be done to determine if stellar structure models including metallicity-dependent rotation can account quantitatively for faster-spinning magnetic neutron stars after core-collapse. If the observed relation shown in Fig. 8 holds true, it would strongly support the magnetar scenario, as it is not clear how the alternative SLSN progenitor models could bring about an equivalent relation between host galaxy metallicity, and light-curve shape and peak luminosity.

We caution that this is a simplistic picture and the spin periods of low-metallicity stars and those of neutron stars formed after collapse are almost certainly affected by other parameters. A dependence on mass is likely and a wide range of ejecta masses have been found (e.g. Nicholl et al. 2015). Stellar binarity and separation distributions will critically affect the final rotation rates of massive stars (de Mink et al. 2013) and hence the spin rates of compact stars. All of these effects are likely to smear out any correlation, hence it is perhaps surprising that we find such a trend. More data may uncover scatter in this plot, or further analytic work may uncover a covariance between parameters that produces the effect. An obvious question to pose is if there is a simple observational parameter that can be plotted which is primarily affected by the spin period (before the

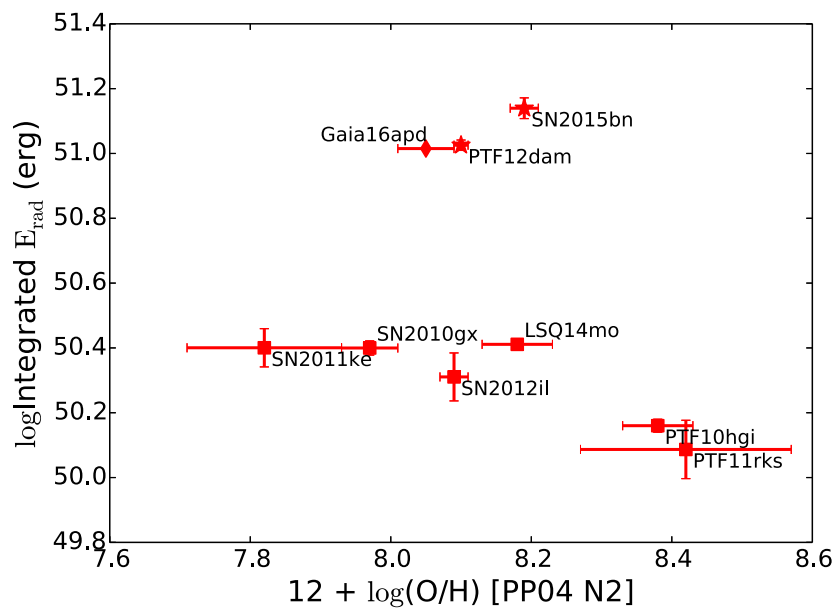

Figure 9. The host metallicity-SLSNe integrated energy comparison. The fast dealing objects to sit on one locus and the slow declining objects to see above, in which the transition object also located.

semi-analytic models produce $\mathrm{P}$ ) and does that show a relation. The three parameters of $\mathrm{B}, \mathrm{P}$ and $\mathrm{M}$ determine the overall shape of the light curve and its luminosity and there is no one single observable that serves as a direct proxy for $\mathrm{P}$.

The only independent observational quantity that it makes sense to test in this way is the total integrated energy. Fig. 9 shows the integrated energy of SLSNe with their host metallicities, which provides a comparison between an observed (rather than model-dependent) SLSN property and the host galaxy. The energy is calculated by integrating a polynomial fit (order 3 ) of the bolometric light curve (in rest-frame) from $\log \left(L_{\max }\right) / \mathrm{e}$, where $\log \left(L_{\max }\right)$ is taken from the fit and $e$ is the neperian number (similar method used in Nicholl et al. 2015). There is an interesting trend for the fast declining objects to sit on one locus and the slow declining objects to sit above. This is perhaps a trivial statement since the slow declining objects stay brighter for longer. However, the integrated energy does not simply represent the spin period, since it is linked to B and to the mass through the spin-down time and diffusion time (see Kasen \& Bildsten 2010).

Pair-instability supernovae (PISNe) also require low metallicity (Langer et al. 2007; Kasen, Woosley \& Heger 2011; Yusof 2013), in order to maintain a sufficiently massive helium core to explode in this manner. However, low metallicity is necessary (but not sufficient in itself) to interpret SLSNe as PISNe. Most Type I SLSNe show light curves that are clearly inconsistent with being PISN (Inserra et al. 2013). The slowly declining events, which have been claimed to be PISN candidates (Gal-Yam et al. 2009; Gal-Yam 2012), seem to have similar hosts to the fast decaying events. They have been interpreted as simply the high-mass counterparts of the fast-declining SLSNe (Nicholl et al. 2013, 2015) and we find no distinction here in their host properties. The slowly declining objects are SN 2007bi, PTF09cnd, PTF12dam, LSQ14an and SN 2015bn and these do not appear significantly different from the rest of the sample in any plots.

\section{CONCLUSIONS}

Comparing the metallicity, sSFR and SN offset for a sample of 19 SLSN Type I host galaxies, we have found perhaps the strongest evidence yet that low metallicity is a key parameter for forming Type I SLSNe. If SLSNe simply followed SFR in the local Universe, 
we would expect to see many more at high metallicity, where the bulk of star formation occurs. Instead, we find that SLSN trace regions of significantly lower metallicity and higher sSFR than those typically found in local galaxies. We suggest that the presence of high SSFR is a consequence of (a) the anticorrelation between gasphase metallicity and sSFR and (b) the requirement of ongoing star formation for massive stellar progenitors to form and to produce SLSNe.

We propose that current evidence supports a metallicity cutoff of about $0.5 \mathrm{Z}_{\odot}$, above which we do not find any Type I SLSNe in our sample. Low metallicity may favour a magnetar (central engine) model, in which reduced mass-loss helps to maintain high angular momentum at core-collapse. We find there is no significant difference of observed host properties among different sub-types of Type I SLSNe. We find a surprisingly clear trend for the derived magnetar spin period (from supernova light-curve fitting) to be correlated with metallicity. The SLSNe from lower metallicity galaxies require magnetars that have shorter spin periods. While this needs further investigation, the correlation supports both the low-metallicity requirement for progenitor stars and the model of magnetar powering.

\section{ACKNOWLEDGEMENTS}

T-WC appreciates Alan Fitzsimmons for thesis advice, and Fabio Bresolin and Rolf Peter Kudritzki for host data collection; thanks to Sandra Savaglio for encouragement and to Dan Perley, Pavel Kroupa and Jeff Cooke for helpful discussions; thanks to the organisers and participants of STScI workshop 'The Mysterious Connection Between SLSNe and GRBs' for stimulating discussions. The research leading to these results has received funding from the European Research Council under the European Union's Seventh Framework Programme (FP7/2007-2013)/ERC Grant agreement no. [291222] (PI : S. J. Smartt). T-WC, RMY and TK acknowledge the support through the Sofia Kovalevskaja Award to PS from the Alexander von Humboldt Foundation of Germany.

\section{REFERENCES}

Angus C. R., Levan A. J., Perley D. A., Tanvir N. R., Lyman J. D., Stanway E. R., Fruchter A. S., 2016, MNRAS, 458, 84

Asplund M., Grevesse N., Sauval A. J., Scott P., 2009, ARA\&A, 47, 481

Bastian N., Covey K. R., Meyer M. R., 2010, ARA\&A, 48, 339

Brott I. et al., 2011, A\&A, 530, A116

Calabrò A. et al., 2017, A\&A, 601, 95

Chabrier G., 2003, PASP, 115, 763

Chen T.-W., 2015, PhD thesis, Queen's University Belfast

Chen T.-W. et al., 2013, ApJ, 763, L28

Chen T.-W. et al., 2015, MNRAS, 452, 1567

Chen T.-W. et al., 2017, A\&A, 602, 9

Cook D. O. et al., 2014, MNRAS, 445, 899

Dabringhausen J., Kroupa P., Pflamm-Altenburg J., Mieske S., 2012, ApJ, 747, 72

de Mink S. E., Langer N., Izzard R. G., Sana H., de Koter A., 2013, ApJ, 764,166

Ekström S. et al., 2012, A\&A, 537, A146

Elmegreen B. G., 2006, ApJ, 648, 572

Fumagalli M., da Silva R. L., Krumholz M. R., 2011, ApJ, 741, L26

Gal-Yam A., 2012, Science, 337, 927

Gal-Yam A. et al., 2009, Nature, 462, 624
Greiner J. et al., 2015, Nature, 523, 189

Hunter I. et al., 2008, A\&A, 479, 541

Inserra C. et al., 2013, ApJ, 770, 128

Jerkstrand A. et al., 2017, ApJ, 835, 13

Kangas T. et al., 2017, MNRAS, 469, 1246

Kasen D., Bildsten L., 2010, ApJ, 717, 245

Kasen D., Woosley S. E., Heger A., 2011, ApJ, 734, 102

Kennicutt R. C., Jr, 1998, ARA\&A, 36, 189

Kennicutt R. C., Jr, Lee J. C., Funes, José G. S. J., Sakai S., Akiyama S., 2008, ApJS, 178, 247

Kobulnicky H. A., Kewley L. J., 2004, ApJ, 617, 240 (KK04)

Langer N., Norman C. A., de Koter A., Vink J. S., Cantiello M., Yoon S.-C., 2007, A\&A, 475, L19

Lee J. C. et al., 2009, ApJ, 706, 599

Leloudas G. et al., 2015b, MNRAS, 449, 917

Lunnan R. et al., 2013, ApJ, 771, 97

Lunnan R. et al., 2014, ApJ, 787, 138

Lunnan R. et al., 2015, ApJ, 804, 90

McCrum M. et al., 2015, MNRAS, 448, 1206

McGaugh S. S., Schombert J. M., 2015, ApJ, 802, 18

Madau P., Dickinson M., 2014, ARA\&A, 52, 415

Mannucci F., Cresci G., Maiolino R., Marconi A., Gnerucci A., 2010, MNRAS, 408, 2115

Marks M., Kroupa P., Dabringhausen J., Pawlowski M. S., 2012, MNRAS, 442, 2246

Martayan C. et al., 2007, A\&A, 462, 683

Massey P., 2011, ASP Conf. Ser., 440, 29

Modjaz M., Kewley L., Bloom J. S., Filippenko A. V., Perley D., Silverman J. M., 2011, ApJ, 731, L4

Mokiem M. R. et al., 2007, A\&A, 473, 603

Moustakas J., Kennicutt R. C., Jr, Tremonti C. A., Dale D. A., Smith J.-D. T., Calzetti D., 2010, ApJS, 190, 233

Neill J. D. et al., 2011, ApJ, 727, 15

Nicholl M. et al., 2013, Nature, 502, 346

Nicholl M. et al., 2014, MNRAS, 444, 2096

Nicholl M. et al., 2015, MNRAS, 452, 3869

Nicholl M. et al., 2016, ApJ, 826, 39

Nicholl M. et al., 2017, ApJ, 835L, 8

Perley D. A. et al., 2016, ApJ, 830, 13

Pettini M., Pagel B. E. J., 2004, MNRAS, 348, L59 (PP04)

Quimby R. M. et al., 2011, Nature, 474, 487

Quimby R. M., Yuan F., Akerlof C., Wheeler J. C., 2013, MNRAS, 431, 912

Ramírez-Agudelo O. H. et al., 2013, A\&A, 560, A29

Salpeter E. E., 1955, ApJ, 121, 161

Sánchez S. F. et al., 2014, A\&A, 563, 49

Schulze S. et al., 2016, preprint (arXiv:1612.05978)

Stoll R., Prieto J. L., Stanek K. Z., Pogge R. W., Szczygieł D. M., Pojmański G., Antognini J., Yan H., 2011, ApJ, 730, 34

Szécsi D. et al., 2015, A\&A, 581, A15

Thöne C. C., de Ugarte Postigo A., García-Benito R., Leloudas G., Schulze S., Amorín R., 2015, MNRAS, 451, L65

Vreeswijk P. M. et al., 2014, ApJ, 797, 24

Woosley S. E., 2010, ApJ, 719, L204

Yates R. M., Kauffmann G., 2014, MNRAS, 439, 3817

Yates R. M., Kauffmann G., Guo Q., 2012, MNRAS, 422, 215

Yoon S.-C., Langer N., Norman C., 2006, A\&A, 460, 199

Young D. R. et al., 2010, A\&A, 512, A70

Yusof N. et al., 2013, MNRAS, 433, 1114

Zahid H. J., Dima G. I., Kudritzki R.-P., Kewley L. J., Geller M. J., Hwang H. S., Silverman J. D., Kashino D., 2014, ApJ, 791, 130

This paper has been typeset from a $\mathrm{T}_{\mathrm{E}} \mathrm{X} / \mathrm{LAT} \mathrm{T}$ file prepared by the author. 